General Conference (Part B)

\title{
THE PERFORMANCE ANALYSIS OF POLARIZATION SHIFT KEYING OPTICAL COMMUNICATION SYSTEM WITH DIFFERENTIAL 4-QUADRATURE SCHEME
}

\author{
Kuen-Suey Hou and Jingshown Wu \\ Room 519 \\ Department of Electrical Engineering and \\ Institute of Communication Engineering \\ National Taiwan University \\ Taipei, Taiwan 10617 \\ R. O.C.
}

\begin{abstract}
The differential 4-quadrature scheme for coherent optical communications is proposed. This system does not need to track the fluctuation of the states of polarizations, which is essential for most other polarization modulation systems. The input message is encoded to the relative position of the present symbol with respect to the reference frame constructed by the previous 3 symbols and the estimated average amplitude of the received symbols is utilized to determine the channel condition. The performance of the particular 4-symbol differential 4-quadrature system is presented.
\end{abstract}

\section{Introduction}

The linearly polarized monochromatic waves propagating along a single mode fiber can be described by two separated orthogonal states of polarizations (SOPs) which provide an additional degree of freedom to carry information for coherent optical communication systems... However, 'the polarization mode coupling and slowly and randomly fluctuation of SOP [1] make these system become very complicated.

Recently, a lot of coherent optical modulation theories are focused on how to efficiently use this degree of freedom. Among them; the Polarization Shift Keying (PolSK) and Stokes Parameters Shift Keying (SPSK) [2-5] use the three 3-dimensional SOPs [6] as the modulation parameters and estimate the SOP fluctuation by introducing an 'adaptive SOP tracking mechanism [3-5]. The Differential PolSK (DPolSK) [7] scheme which eliminates the SOP tracking mechanism, provides another choice with some degradation in performance.

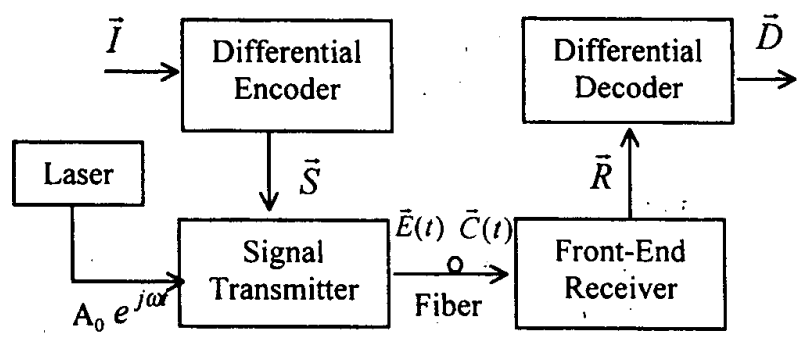

Fig.1 The block diagram of the D4Q system

On the other hand, the 4-quadrature [8-9] system is proposed to recover the original information in two orthogonal SOPs by the reference vector estimator which is assumed to adaptively and perfectly track the random drift of the channel Jones matrix [1]. The signal symbols can be represented by the 4dimensional vector, and the sensitivity of the system is dramatically improved, especially at higher order constellations.

In this paper, we'll show that if neither the Jones matrix estimation nor Phase Locked Loop (PLL) is applied, we may encode the information in the relative position of the present symbol with respect to the previous three symbols. At the receiver, we only need to estimate the amplitude which is much simpler than to constantly evaluate the Jones matrix [8].This scheme is named as the differential 4 quadrature (D4Q) modulation.

\section{System description}

Fig. 1 : shows the system diagram. In the differential encoder, the information vector, $\vec{l}$, is translated into the unit 4-dimensional symbol vector $\vec{S}=\left[s_{j}, s_{2}, s_{3}\right.$, 




Fig.2 The structure of the D4Q front-end receiver, where I\&D means the integrate and dump circuits

$\left.s_{4}\right]^{\top}$ which is modulated into the transmitting signal $\vec{E}(t)$, where $\vec{E}(t)$ represents the electrical field of the lightwave carrier. After propagating through the fiber, the received electrical field of the lightwave at the receiver is expressed by $\vec{C}(t)$. The structure of the D4Q front-end receiver, as shown in Fig. 2, includes a local laser, two polarization beam splitters (PBS), and two $90^{\circ}$ hybrids (HY) which split the received lightwave $\vec{C}(t)$ and the local laser into four branches followed by the heterodyne detector and the IF signal space projection circuits. The heterodyne detector consists of a photo-detector and an intermediate frequency filter (IFF), which converts the received lightwave into the corresponding electrical IF signal. The IF signal space projection circuit is made up of a correlator, which demodulates and translates the IF signal $\vec{w}(t)$ to a baseband 4dimensional vector $\vec{R}$. Here the local IF oscillator signal is generated by an Automatic Frequency Control (AFC) loop [10]. Finally, the received vector $\vec{R}$ is decoded into the estimated information vector $\vec{D}$ at the differential decoder.

In general, when the data rate is much larger than the laser linewidth, laser phase noise can be neglected [11]. Such that we can assume that the frequency of the local IF oscillator is exactly the same as the intermidiate frequency, $\omega_{I F}$, with a constant phase Shift $\varphi$. Following the normalization conventions, the IF filter noise bandwidth is equal to twice of the symbol rate $R_{s}[12-13]$ and we can derive the received vector as

$\vec{R}=\mathrm{A} \mathbf{H}_{\mathrm{T}} \vec{S}+\vec{n}_{c}=\mathbf{H}_{\mathrm{T}}(\mathrm{A} \vec{S}+\vec{n})$

where $A$ is the normalized received signal amplitude [4], $\mathbf{H}_{\mathbf{T}}$ is a unitary matrix, both $\vec{n}_{c}$ and $\vec{n}$ are vectors whose elements are independent identically distributed (i.i.d.) Gaussian noises with zero mean and variance $\sigma^{2}=R_{s}$.

Because the operations of encoding and decoding involve several consecutive signals, we will have subscript $k$ in the notations to represent the signals in the time slot $k$. At the transmitter, we can denote the consecutive transmitting vectors as $\mathrm{A}_{0} \vec{S}_{n-3}, \mathrm{~A}_{0} \vec{S}_{n-2}$, $\mathrm{A}_{0} \vec{S}_{n-1}$, and $\mathrm{A}_{0} \vec{S}_{n}$ [11], where $\mathrm{A}_{0}$ is the amplitude of the lightwave carrier at the transmitter. At the outputs of the front-end receiver, the corresponding signals are $\vec{R}_{n-3}, \vec{R}_{n-2}, \vec{R}_{n-1}$, and $\vec{R}_{n}$. The differential decoder output is $\vec{D}_{n}$. In order to have the system operate properly, any three consecutive vectors $\vec{S}_{k}, \vec{S}_{k+1}$, and $\vec{S}_{k+2}$ must be linearly independent.

At the transmitter the differential encoder encodes the information vector $\overrightarrow{\mathrm{L}}_{\mathrm{n}}$ into $\overrightarrow{\mathrm{S}}_{\mathrm{n}}$ by

$\overrightarrow{\mathrm{S}}_{\mathrm{n}}=\mathbf{M} \overrightarrow{\mathrm{L}}_{\mathrm{n}}$

where $\mathbf{M}$ is the matrix $\left[\overrightarrow{\mathrm{u}}_{1}, \overrightarrow{\mathrm{u}}_{2}, \overrightarrow{\mathrm{u}}_{3}, \overrightarrow{\mathrm{u}}_{1} \times \overrightarrow{\mathrm{u}}_{2} \times \overrightarrow{\mathrm{u}}_{3}\right],\left(\overrightarrow{\mathrm{u}}_{1}\right.$, $\left.\vec{u}_{2}, \vec{u}_{3}\right)$ is a set of orthonomal vectors spanning the same space as $\left(\overrightarrow{\mathrm{S}}_{n-3}, \vec{S}_{n-2}, \overrightarrow{\mathrm{S}}_{n-1}\right)$, and is extracted by some specific basis-constructing operation $\mathbf{N}$, such as Gram-Schmidt process [14]. Therefore we can denote ( $\overrightarrow{\mathrm{u}}_{1}, \overrightarrow{\mathrm{u}}_{2}, \overrightarrow{\mathrm{u}}_{3}$ ) as $\mathbf{N}\left(\overrightarrow{\mathrm{S}}_{\mathrm{n}-3}, \overrightarrow{\mathrm{S}}_{\mathrm{n}-2}, \overrightarrow{\mathrm{S}}_{\mathrm{n}-1}\right)$, and $\overrightarrow{\mathrm{u}}_{1} \times \overrightarrow{\mathrm{u}}_{2} \times \overrightarrow{\mathrm{u}}_{3}$ is so-called generalized cross product [14].

$\mathbf{M}$ can be proved to be unitary. Notice that the element vectors of $\mathbf{M}$ are the basis derived from $\vec{S}_{n-3}, \vec{S}_{n-2}$, and $\vec{S}_{n-1}$. Similarly, the output of the differential decoder $\vec{D}_{n}$ can be represented by $\vec{R}_{n}$ and the set of basis $\vec{v}_{1}, \vec{v}_{2}, \vec{v}_{3}$ and $\vec{v}_{1} \times \vec{v}_{2} \times \vec{v}_{3}$ which 


\begin{tabular}{|c|c|c|c|c|}
\hline $\overrightarrow{\mathrm{I}}_{\mathrm{n}}$ & $\overrightarrow{\mathrm{e}}_{1}$ & $-\overrightarrow{\mathrm{e}}_{1}$ & $\overrightarrow{\mathrm{e}}_{4}$ & $-\overrightarrow{\mathrm{e}}_{4}$ \\
\hline Bit coding & 00 & 11 & 01 & 01 \\
\hline
\end{tabular}

Table 1 Four possible information vectors $\vec{I}_{n}$ 's and the corresponding bit codings

are extracted from $\vec{R}_{n-3}, \vec{R}_{n-2}$, and $\vec{R}_{n-1}$ by the operation $\mathbf{N}$. That is,

$\vec{D}_{n}{ }^{\top}=\left[d_{1}, d_{2}, d_{3}, d_{4}\right]=\vec{R}_{n} \cdot\left[\vec{v}_{1}, \vec{v}_{2}, \vec{v}_{3}, \vec{v}_{1} \times \vec{v}_{2} \times \vec{v}_{3}\right]$

Notice that $\vec{R}_{n}$ and $\vec{S}_{n}$ are related as Equation (1) indicates. When the Gram-Schmidt process is used, we can show that in the noiseless channel, the information vector can be totally recovered.

The amplitude value of the received vector is indispensable in the calculation of the Gram-Schmidt process, such that we have to employ the automatic gain control (AGC) technique in the system. For practical consideration, the received symbol includes noise terms. But we can expect that the additional noise only induces a little perturbation terms, when the signal to noise ratio is large enough. Of course, how the noise terms influence the system performance requires more rigorous treatment. As an example, a special 4-symbol D4Q (4s-D4Q) system will be described and its noise performance is analyzed.

The 4s-D4Q system originates from the 8-symbol 4Q system with the constraint that any consecutive 3 symbols are linearly independent. The 4 possible information vectors $\vec{I}_{n}$ 's and the corresponding bitcodings are shown in Table 1 , where we define $\overrightarrow{\mathrm{e}}_{1}=\left[\begin{array}{llll}1 & 0 & 0 & 0\end{array}\right]^{\mathrm{T}}, \overrightarrow{\mathrm{e}}_{2}=\left[\begin{array}{llll}0 & 1 & 0 & 0\end{array}\right]^{\mathrm{\top}}, \overrightarrow{\mathrm{e}}_{3}=\left[\begin{array}{llll}0 & 0 & 1 & 0\end{array}\right]^{\mathrm{\top}}$, and

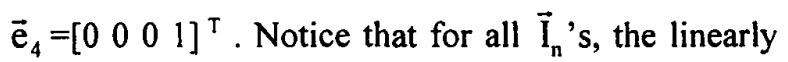
independent constraint of $\overrightarrow{\mathrm{S}}_{k}, \overrightarrow{\mathrm{S}}_{\mathrm{k}+1}$, and $\overrightarrow{\mathrm{S}}_{\mathrm{k}+2}$ is always satisfied. The encoding scheme is the same as Equation (2), but the basis-constructing operation, $\mathbf{N}$, is simplified as follows:

$\mathbf{N}\left(\vec{S}_{n-3}, \vec{S}_{n-2}, \vec{S}_{n-1}\right)=\left(\vec{S}_{n-3}, \vec{S}_{n-2}, \vec{S}_{n-1}\right)$

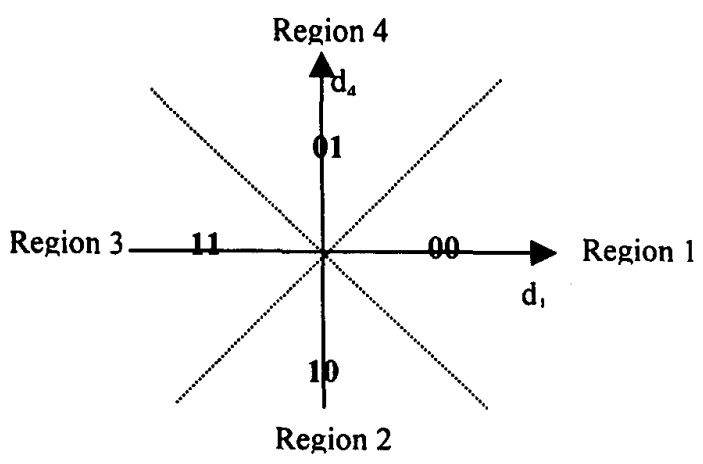

Fig.3 The decision regions for the 4s-D4Q system

And

$\mathbf{N}\left(\overrightarrow{\mathbf{R}}_{n-3}, \overrightarrow{\mathbf{R}}_{n-2}, \vec{R}_{n-1}\right),=\left(\vec{R}_{n-3}, \vec{R}_{n-2}, \vec{R}_{n-1}\right) / A \ldots(5)$

where $A$ is assumed to be well estimated at the receiver by the $A G C$ mechanism.

The decoding procedure is the same as shown in the previous section, and the estimated information vector is given as

$\vec{D}_{n}{ }^{\top}=\vec{R}_{n} \cdot\left[\vec{R}_{n-3}, \vec{R}_{n-2}, \vec{R}_{n-1},\left(\vec{R}_{n-3} \times \vec{R}_{n-2} \times\right.\right.$

$\left.\left.\vec{R}_{n-1}\right) / A^{2}\right] / A=$

$\left(A \vec{I}_{n}+\vec{n}_{4}\right) \cdot\left[A \vec{e}_{1}+\vec{n}_{1}, A \vec{e}_{2}+\vec{n}_{2}, A \vec{e}_{3}+\vec{n}_{3}\right.$,

$\left.\left(A \vec{e}_{1}+\vec{n}_{1}\right) \times\left(A \vec{e}_{2}+\vec{n}_{2}\right) \times\left(A \vec{e}_{3}+\vec{n}_{3}\right) / A^{2}\right] / A \ldots .(6)$

where $\vec{n}_{i}, \mathrm{i}=1,2,3$, and 4 , are vectors whose elements are i.i.d Gaussian noise with zero mean and variance $\sigma^{2}=R_{s}$.

Equation (6) means the estimated information vector $\vec{D}_{n}$ is only a little perturbed as assumed. Notice that $\vec{D}_{n}$ is irrelevant to the channel Jones matrix and the IF frequency phase difference $\varphi$. In other words, the output of the differential decoder can be deduced from the original signal $\vec{I}_{n}$ and the received signal amplitude without knowing the exact SOP of the lightwave carrier. Therefore, the polarization SOP fluctuation can be totally ignored. 


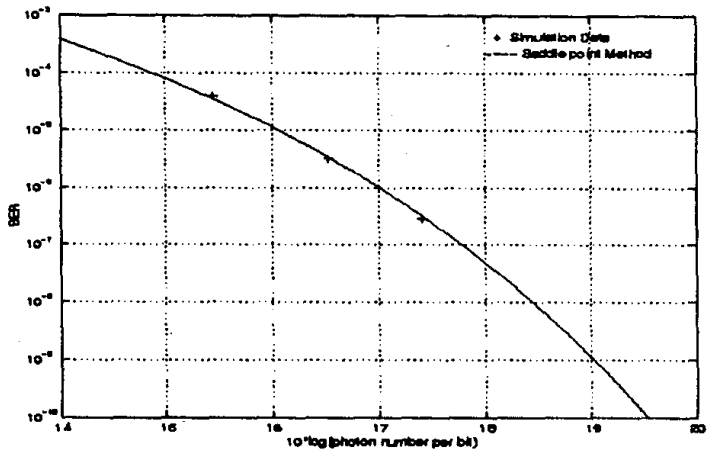

Fig.4 BER performance, for the case $\vec{I}_{n}=\vec{e}_{4}$

\section{Performance analysis of the 4s-D4Q system}

When the decision regions are taken as in Fig.3, we can analyze the bit error rate (BER) by applying the saddle point approximation method [15]. For the case $\vec{I}_{n}=\vec{e}_{4}$, the analytic result is given as

$\operatorname{BER}\left(\overrightarrow{\mathrm{I}}_{\mathrm{n}}=\overrightarrow{\mathrm{e}}_{4}\right) \sim \mathrm{Q}(\sqrt{\mathrm{F} / 4.44})$

where $Q($.$) is the probability Q-function, and F$ is the number of photons per symbol. Fig.4 depicts the BER of the analytical and simulation results.

Other cases can be done in the similar way, and the approximation and simulation results also agree with each other very well. Assume that all symbols are equally probable, we have the mean BER as follows:

Mean BER $\{\mathrm{Q}(\sqrt{\mathrm{F} / 4.44})+\mathrm{Q}(\sqrt{\mathrm{F} / 4.12})\} / 2 \ldots \ldots$. (8)

The result is ploted in Fig. 5. Notice that the horizontal axis is the number of photon per bit. In the $4 \mathrm{~s}-\mathrm{D} 4 \mathrm{Q}$ system, it is equal to $\mathrm{F} / 2$.

\section{Conclusions}

The theory and the structure of the differential 4 quadrature $(D 4 Q)$ system for the coherent optical communication is proposed first time. The D4Q system, which encodes the information in the relative position of the consecutive 4 symbols, doesn't need

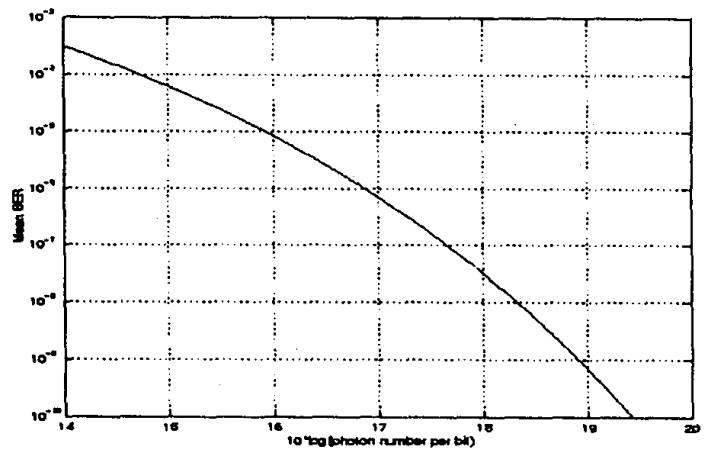

Fig.5 The mean BER performance of the 4s-D4Q system

to constantly track the random drift of the channel Jones matrix, and has no phase ambiguity problem which is unavoidable in the coherent phase shift keying system. As an example, the 4s-D4Q system constructed in a simpler way is presented and analyzed. Because the symbols of $D 4 Q$ systems are scattered over a 4-dimensional space instead of the conventional two-dimensional one, such that we expect the D4Q system will perform better at higher order constellation, as is the case in the 4 quadrature (4Q) system.

\section{Acknowledgement}

The authors would like to appreciate the support from the National Science Council under the grant NSC 88-2213-E-002-081, and valuable discussions with Mr. Meng-guang Tsai.

\section{References}

[1] S. C. Rashleigh, "Origins and control of the polarization effects in single-mode fibers," $J$. Lightwave Technol., Vol. 1, no.2, pp.312-321, 1983

[2] S. Benedetto and P. Poggiolini, "Theory of polarization shift keying modulation," IEEE Trans. Commun., Vol.40, no.4, pp.708-721, Apr. 1992

[3] S. Benedetto and P. Poggiolini, "Multilevel polarization shift keying: optimum receiver structure and performance evaluation," IEEE Trans. Commun., Vol.42, no.2/3/4, pp.1174-1186, 1994 
[4] S. Betti, F. Curti, G. D. Marchis, and E. Iannone, "Multilevel coherent optical system based on Stokes parameters modulation," J. Lightwave Technol., Vol.9, no.10, pp.1314-1320, Oct.1991

[5] S. Benedetto, R. Gaudino, and Poggiolini, "Polarization recovery in optical polarization shift keying system," IEEE Trans. Commun., Vol.45, no.10, pp.1269-1279, Oct. 1997

[6] M. Born and E. Wolf, Principles of Optics, Oxford, England: Pergamon, 1975

[7] R. J. Blaikie, D. P. Taylor, and P. T. Gough,"Multilevel Differential Polarization Shift Keying," IEEE Trans. Commun., Vol. 45, No.1, pp.95-102, 1997

[8] S. Betti, F. Curti, G.D. Marchis, and E. Iannone, "A novel multilevel coherent optical system: 4quadrature signaling", J. Lightwave Technol., Vol. 9, No. 4, pp.514-523, 1991

[9] S. Betti, G. Marchis, E. Iannone, "Toward an optimum use of the optical channel capacity," Fiber and Integrated Optics, Vol.13, no.2, pp.147-164, 1994

[10] S. Haykin, Communication Systems, Third edition, New York: John Wiley and Sons, 1994

[11] Max M. K. Liu, Principles and applications of optical communications, Chicago: Irwin, 1996

[12] J. Salz, "Coherent lightwave communications," AT\&T Technol. J., Vol.64, no.10, pp.2153-2209, Dec 1985

[13] G. Einarsson, Principles of Lightwave Communications, New York: John Wiley and Sons, 1995

[14] David M. Bloom, Linear Algebra and Geometry, New York: Cambridge University Press, 1979

[15] N. Bleistein and R. A. Handelsman, Asymptotic expansions of Integrals, New York: Dover Publications, 1986 